\title{
The Dynamic Theory of Macro-systems Usage to Solving the Problems of Transportation Processes Efficiency Increasing
}

\author{
Igor Agureev ${ }^{1}{ }^{*}$, Vladislav Pyshnyi ${ }^{1}$, Vitalii Mityugin ${ }^{1}$, and Nukolai Frolov ${ }^{1}$ \\ ${ }^{1}$ Tula State University, 92 Lenina prosp., Tula, 300012, Russia
}

\begin{abstract}
The transport system of the city (agglomeration) is complex, and consists of a set of stochastic functioning elements. It allows choosing the theory of macro-systems, which investigates appearance of the determined behavior at the level of "system as a whole" as the main theoretical basis. The theory in this paper extends for the transport systems dynamic models development and separate processes or elements of them. Thus, there is an opportunity to find not only equilibrium conditions of systems, but also transition processes. Separate elements can have the properties of so-called "active particles", the plenty of which creates conditions to the cooperative behavior.
\end{abstract}

\section{Introduction}

Any transport system of the city (or agglomeration) is a complex system, in which a set of processes relating to planning, the organization and performance of transportation of goods and passengers, to monitoring and quality control, availability of transportations, etc. is going on. Any of these aspects contains the practical problems depending on the region specifics.

Research and regulation of spatial structures of the cities is the actual direction of the theory and practice of city development [12]. It is known that a city includes the number of subsystems, such as a place of employment, housing, service, etc., and also infrastructure (transport, communication, safety, etc.) providing material and information flows between the elements of an urban environment. It is known also that not only the cities and megalopolises, but also averages and even small settlements cannot solve all the problems without use of the external territories adjacent to borders of cities [1]. Thus, there are inevitably the similar tasks for a city agglomeration as a whole together with its center during research and regulation of city systems and their spatial structures.

Let's note the main assumptions and theoretical bases used at a solution of the specified tasks relying on the most fundamental results in the field of the theory of macro-systems [12, 16]. It is necessary to specify the macro-systems as such object, in which the stochastic behavior of its elements is able to be transformed to the determined behavior of system as a whole. The urban environment has to be considered in development taking into account dynamics. Thus the models of various subsystems are allocated in the functional and spatial

*Corresponding author: agureev-igor@yandex.ru 
model of the city system. The necessary element is existence of the system regulators providing management of a development of a city. The models of a transport subsystem constructed on the basis of macroscopic approach are the most important $[5,13]$.

\section{Material and methods}

Macroscopic and microscopic approaches are often used the concepts of the different areas of mathematical modeling. If we discuss about modeling of transport systems both approaches are known in the theory of transport flows. However, they are quite applicable in a wider scale, for example, at the description of a transport subsystem of the city (or agglomeration) in general. We talk about "macroscopic approach" if the mathematical model abstracts from the description of each element. The concept of "microscopic approach" is realized when "the movement equations" for each separate element are used in the model. Thus, it can significantly be specified how the separate element behaves at the level of microscopic description. It is necessary to distinguish two cases: 1) stochastic behavior of elements (elements with casual behavior); 2) the determined behavior of elements. Nevertheless, both in the first, and in the second case emergence of extremely complex dynamics is possible in system as a whole.

For the first case it is possible to refer as an example to variants of models of transport queuing systems describing some casual processes of receipt of system elements on an entrance of service channels. For the second case it is possible to specify the known class of models of "following for the leader" in the transport flows theory.

Further generalization of collective behavior of separate elements within the traditional theory of macro-systems often leads to emergence of the determined state of system as a whole. However, it is worth to remember that in systems with the determined behavior of separate elements possibly emergence of chaotic behavior of the system. Thus, we have to point to expansion of positions of the theory of macro-systems, having expressed it as the dynamic theory of transport macro-systems. The dynamic system analysis in conjunction with macroscopic approach is the methodical base of optimum planning and procedures of decision-making in the field of functional and spatial development of city systems [9]. Thus, the main direction of development of the transport macro-systems theory is seen in broad attraction of open systems physics [6,14] and the theory of active particles [10].

\section{Theory. Bases of the dynamic theory of transport macro- systems}

Let's consider [11] the generalized transport macro-system with continuous time $t$ and containing $Y$ same elements with some type of behavior from a set $B(t)=\left\{\beta_{d}(t), \beta_{s}(t)\right\}$. Let each element can have a state from $\rho$ classes $\mathrm{K}_{1}, \ldots \mathrm{K}_{\rho}$. Classification of states such is that these classes don't crossed.

Let's note $\sigma^{1}, \ldots \sigma^{\rho}$ - sets of states, where $\sigma^{i} \in \mathrm{K}_{i}$. Let's further believe that sets of states discrete for $\beta_{S}(t)$ (or continuous for $\beta_{d}(t)$ ) and contain the finite number of elements (or infinite respectively).

In the present paper we will be limited to a case of a uniform macro-system, which elements can accept states only of one class. For example, it is possible to understand as a class of states "an attachment in the transport area or zone" (an outlet-inlet of system elements). This class of states can be applied to elements with behavior type $\beta_{s}(t)$. The "average speed of a transport flow in a road span" applied to "vehicle" or "transport flow" elements with the determined behavior type $\beta_{d}(t)$ can be another example of the concept 
"state class". To such class the continuous (infinite) set of states connected by the determined manner with sets of states of other classes corresponds. Thus, systems with the determined type of behavior within the accepted definition cannot be uniform as are connected along with several classes of states.

So, the uniform system has one set of states of elements $\sigma$, which subsets $\sigma_{1}, \ldots \sigma_{m}$ are such that their association coincides with $\sigma$, and the crossing of any couples is empty.

Elements of a macro-system can incidentally and independently from each other get to any state from subsets $\sigma_{1}, \ldots \sigma_{m}$. Concerning each fixed subset $\sigma_{n}$ it is two opportunities for an element: to get to any state from $\sigma_{n}$ with aprioristic probability $a_{n}$ and not to get with probability $\left(1-a_{n}\right)$.

Let's consider as an example some set of elements of a transport macro-system of automobile (city) transport, which can be in the corresponding states (table 1).

Table 1. Elements and states of a transport macro-system

\begin{tabular}{|c|c|c|}
\hline № & Elements & States \\
\hline 1 & Vehicle (V) & \multirow{7}{*}{$\begin{array}{l}\text { a. The element } i \text { is in the transport region of } j \text {; } \\
\text { b. The element } i \text { is in the transport region of } j \text { and } \\
\text { a zone of the transport region of } k \text {; } \\
\text { c. The element } i \text { is in a stage (span) of } j \text { of the } \\
\text { street road network (SRN); } \\
\text { d. The element } i \text { is in the } j \text { vehicle; } \\
\text { e. The element } i \text { is on } j \text { route; } \\
\text { f. The element } i \text { is on a stopping point of } j \text {, etc. }\end{array}$} \\
\hline 2 & Route Vehicle (RV) & \\
\hline 3 & Not-route Vehicle (NRV) & \\
\hline 4 & $\begin{array}{c}\text { Driver of the Vehicle } \\
\text { (DV) }\end{array}$ & \\
\hline 5 & Passenger & \\
\hline 6 & Pedestrian & \\
\hline 7 & Route & \\
\hline
\end{tabular}

Table 1 contains basic elements and states, which can be supplemented depending on the maintenance of a task. The task definition, at which there are all elements (and the sets of states corresponding to them), is hardly expedient and possible in principle from the point of view of its resolvability. More rational way is the formulation of special cases, which contain no more than 2-3 elements. For example, it is possible to consider the solution of a task on filling of routes by vehicles taking into account fullness. Then in a task definition elements 2,5,7 and the corresponding possible states $\{\mathbf{d}, \mathbf{e}, \mathbf{f}\}$ will be used.

The theory of macro-systems has the main stages of development, which we specify in according to the Yu. S. Popkov's works [11, 12]: 1) terms and concepts, the phenomenological schemes are entered; 2) the basic concept "probability of a macro-state" as the value $P(N)=\prod_{n=1}^{m} P_{n}\left(N_{n}\right)$, which is expressed through probabilities of states of separate elements $n=1, \ldots m$, is entered; 3$)$ to define the function $P(N)$ the mechanism of filling of states in $\sigma_{n}$ depending on type of states is considered (Fermi-, Einstein-, or a Boltzmann-state), and on this basis the function $P(N)$ is defined as physical (or information) entropy; 4) the received probabilistic characteristics allow to consider the features of the phenomenological schemes of the uniform isolated macro-system consisting in not equiprobable choice elements of system of the corresponding states in subsets $\sigma_{n}(n \in \overline{1, m})$; 5 ) the conclusion that the generalized entropy for a macro-system has the only maximum which "sharpness" with growth of number of elements increases in system is done; 6) distribution of elements of uniform system on subsets of close states which can be connected, for example, with an expenditure of various resources, is established by existence and action of "price functions" of a transport network, etc., that is the solution of the specific objectives connected most often with determination of balance of a macro-system is carried out. An example of such task is classical statement about flows of a street road network [14, 15] in which entropy approach at the accounting of distribution of range of trips is used [16]. 
Among the main shortcomings of the traditional theory it is necessary to specify lack of opportunities for the accounting of processes in open systems, and also impossibility of consideration of dynamical processes.

The dynamic theory of transport macro-systems can be developed on the basis the following basic assumptions.

- The phase space "the generalized coordinates - the generalized impulses" is entered. Dimension of space depends on conditions of specific objectives.

- It is supposed that the phase space has a function of distribution of elements on "coordinates" and "impulses", which depends on time.

- Existence of the generalized kinetic equation (like Boltzmann's equation) is postulated if justice of a thermodynamic approach is established for the considered macro-system (possibility of the description of a macroscopic state of system, several parameters of which are consisting of a large number of elements).

- It is supposed that macro-system carries out temporary evolution, which can be characterized by change of the degree of randomness with the entropy of a non-equilibrium process. The entropy is expressed through the distribution function entered above.

- Change of the degree of randomness of a system is one of the basic concepts of the dynamic theory of transport systems, which is absent in an explicit form in the traditional theory of macro-systems. The analog of the H-theorem of Boltzmann for the externally closed systems is established to claim that during temporary evolution to an equilibrium state the entropy of a system increases and remains invariable at its achievement.

- For the open transport macro-systems, which are in states of continuous interaction with the systems of a higher level, existence of the analog of "energy", possibility of change of the degree of randomness during evolution and use of the basis of physics of open systems, in particular Klimontovich's S-theorem, are postulated [7].

For the accounting of dynamics in macro-systems the concept "active particle" is entered, and then there are possibilities of application of results of the corresponding theory [10]. In particular, it gives the chance for transport systems to consider more fully probabilistic features of behavior of such particles, as which any element in table 1 can act.

\section{Results. Examples of the solved tasks}

Representing vehicles and goods as "active particles" within the corresponding theory [10] it is possible to consider a kinetic picture of collective behavior of a macro-system by means of logistic system model. The variant can be presented by the equations:

$$
\left\{\begin{array}{l}
\dot{x}=a[(X-x)+k y-m z](Z-z)-b(Y-y)(Z-z) \\
\dot{y}=c[(Y-y)+l x+n z] z-d(X-x) z \\
\dot{z}=e x-f y+g .
\end{array}\right.
$$

Here the variables have the following sense: $x$ - the cars delivering freight into a warehouse; $y$ - the cars carrying freight from a warehouse; $z$ - quantity of freight in a warehouse. Parameters in the equations (1) note: $X$ - number of the cars participating in delivery of freight; $Y$ - number of the cars participating in the carrying of freight; $Z$ - limit (or the most probable value) warehouse capacity; $g$ - intensity of completion of freight by other types of transport. Such systems are the subject of consideration of nonlinear dynamics and the theory of catastrophes [17]. 


\section{Discussion}

The system of the equations (1) decided at different parameters by the Runge-Kutta method with a variable step and accuracy of the order $1 \cdot 10^{-6}$. All main types of the attractors (a focus, a limit cycle, or an irregular attractor) were found in the model. Some types of complex dynamics are presented to models (1) (see table 2). These results testify that rather simple equations of the model of a macro-system can show complex dynamics and at the macroscopic level can be observed not only an equilibrium state, but also chaotic fluctuations in the vicinity of these states.

\section{Conclusion}

In the present paper the examples of city transport systems (or agglomerations) with complex dynamics are shown. Such systems consist of a set of stochastic functioning elements, but their cooperative behavior transforms to deterministic phase trajectories. It allowed to choose the theory of transport macro-systems, which investigates emergence of the determined behavior at the level of "system in general", as the main theoretical basis. The found faults of the traditional theory, such as lack of opportunities for the accounting of processes in open systems, and also impossibility of consideration of processes in dynamics, stimulated to expand basic provisions of the theory for the purpose of development of dynamic models of transport systems and their separate processes or elements [8, 2, 3, 4]. Thus there is an opportunity to find not only equilibrium states of systems, but also transition processes. Separate elements can have properties of so-called "active particles" the set of which creates conditions of the coordinated behavior.

The results of the research project are published with the financial support of Tula State University within the framework of the scientific project № 2017-82.

Table 2. Complex dynamics in logistic system

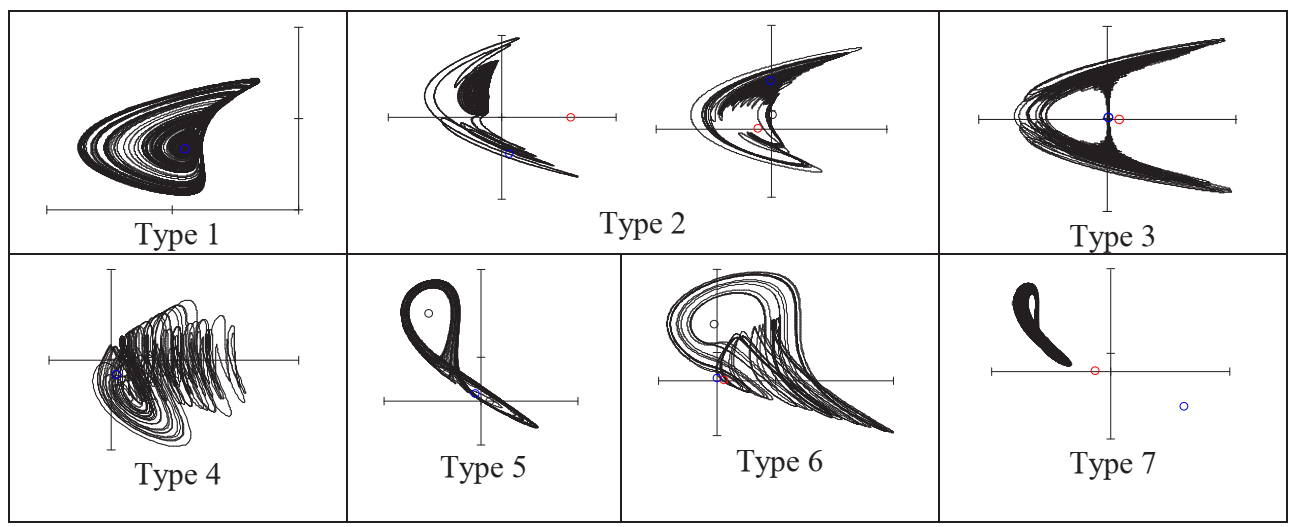

\section{References}

1. A.A. Agasyants, Set of Automobile Highways in the Largest Cities. Transport and town-planning problems. M.: Publishing house of DIA; 248 p. (2010)

2. I. E. Agureev, E. E. Atlas, The Chaotic Dynamics in Systems of Transport. Complexity. Reason. Postneklassika. No. 1, P. 94-106. (2010) 
3. I.E. Agureev, E.E. Atlas, N.S. Pastukhova, The Chaotic Dynamics in the Mathematical Models of Transportation Systems. Izvestiya of the Tula State University.

Tekhnicheskie nauki. № 3, P. 372-389. (2012)

4. I. E. Agureev, M. Yu. Elagin, V. A. Pyshnyi, R. N. Khmelev, Methodology of Substantiation of the City Transport System Structure and Integration of Intelligent Elements into it. Transportation Research Proc. Ser. "12th International Conference "Organization and Traffic Safety Management in Large Cities" SPbOTSIC 2016". P. 813. (2017)

5. J. Holngren, et al. Combining macro-level and agent-based modeling for improved freight transport analysis. 5th International Conference on Ambient Systems, Networks and Technologies (ANT-2014). Procedia Computer Science. 32. P. 380-387. (2014)

6. Introduction into Mathematical Modeling of Transport Flows // Gasnikov A. V., etc. Editor A. V. Gasnikov. M. Publishing house of MTsNMO; 2013, 427 p.

7. Yu.L. Klimontovich, Introduction to Physics of Open Systems. M.: Publishing house Janus; 2002, 284 p.

8. V.A. Korchagin, S.A. Lyapin, A.N. Novikov, J.N. Rizayeva, Complex self-developing transport system. Mir transporta i tekhnologicheskih machin. №2 (53), p.110-116. (2016)

9. A.N. Novikov, V.A. Golenkov, Yu.N. Rams, A.A. Katunin, A.S. Bodrov Improvement of a Road Network for Increase of their Flow Capacity and Use of Means of Transport Telematics. Izvestiya of the Tula State University. 2014, No. 6, P. 128-139.

10. A.I. Olemskoi, The Sinergetics of the Complex Systems: Phenomenological and Statistical Theory. M.: Publishing house KRASAND; 384 p. (2009)

11. Yu.S. Popkov, The Theory of Macro-systems (The Models of Equilibrium). M.: Editorial URSS; 320 p. (1999)

12. V.N. Resin, Yu.S. Popkov, Development of Cities in the Conditions of a Transitional Economy. M.: Editorial URSS; 328 p. (2000)

13. K. Solecka, J. Zak, Integration of the urban public transportation system with the application of traffic simulation. Transportation Research Proc. 17th Meeting of the EURO Working Group on Transportation, EWGT2014, 2-4 July 2014, Sevilla, Spain. 3. P. 259-268. (2014)

14. V. I. Shvetsov, Mathematical Modeling of Traffic Flows. Automation and Remote Control. V. 64. № 11. P. 1651-1689. (2003)

15. V. I. Shvetsov, Algorithms for Distributing Traffic Flows. Automation and Remote Control. V. 70. № 10. P. 1728-1736. (2009)

16. A.G. Wilson, Entropy in Urban and Regional Modelling, Pion Press, London. (1970)

17. A.G. Wilson, Catastrophe Theory and Bifurcation; Applications to Urban and Regional Systems, University of California Press, Berkeley, CA. (1981) 